\title{
Scaffold Proteins in Gastrointestinal Tumors as a Shortcut to Oncoprotein Activation
}

\author{
Chushu Li Huanbin Wang Han Yao Jing-Yuan Fang Jie Xu \\ Division of Gastroenterology and Hepatology, Renji Hospital School of Medicine, Shanghai \\ Jiao Tong University, Shanghai, China
}

\section{Keywords}

Gastrointestinal tumors · Mitogen-activated protein kinase $\cdot$ p53 $\cdot$ Scaffold proteins

\begin{abstract}
Background: The development of cancer involves uncontrolled cell proliferation, and multiple signaling pathways that regulate cell proliferation have been found to be dysregulated in cancers. Extracellular signal-regulated protein kinase (ERK) is one of three major subtypes in the mitogen-activated protein kinase (MAPK) families. The MAPK/ERK pathway (RAS/RAF1/ MEK/ERK) plays an important part in promoting cell proliferation in response to growth factors, thereby serving as a driving signal in gastrointestinal (GI) tumors. In contrast, the p53 tumor suppressor functions as a "guardian of the genome" and stops cell proliferation when oncogenic signaling is activated. Summary: Both pathways constrain each other in healthy GI epithelium, facilitating controlled proliferation that is essential for tissue repair and regeneration. However, in GI tumors, the MAPK/ERK and p53 pathways are commonly dysregulated, in part due to abnormal posttranslational modifications. Hyperphosphorylation of the ERK protein causes sustained activation of cell proliferation, whereas hypoacetylation of the p53 protein impairs its transcriptional function and blocks cell apoptosis. Multiple scaffold proteins have been found to regulate the posttranslational modifications of ERK and p53 proteins in GI tumors. Key Message: Abnormal expression of scaffold proteins may contribute to the dysregulation of the MAPK and p53 signaling pathways and thereby contribute to the development of GI tumors. Practical Implications: Scaffold proteins are potential biomarkers and therapeutic targets in GI tumors.

(c) 2017 S. Karger AG, Basel
\end{abstract}

Jing-Yuan Fang or Jie Xu

Division of Gastroenterology and Hepatology

Renji Hospital School of Medicine, Shanghai Jiao Tong University

Shanghai 200001 (China)

E-Mail jingyuanfang @ sjtu.edu.cn or jiexu@ sjtu.edu.cn 
Li et al.: Scaffold Proteins in Gastrointestinal Tumors as a Shortcut to Oncoprotein Activation

\section{Mitogen-Activated Protein Kinase Signaling Plays Crucial Roles in Gastrointestinal Tumors}

The human genome encodes three major subtypes of mitogen-activated protein kinases (MAPKs): extracellular signal-regulated protein kinase (ERK), c-jun N-terminal kinase, and MAPK14. In a previous work, we systematically discussed the roles of MAPK pathways in colorectal cancer [1]. The ERK signaling pathway plays a central role in regulating cell proliferation as well as triggering transcription factor activation and histone modification in response to growth factor stimulation. A phosphorylation cascade is transduced from the small guanosinetriphosphatase (GTPase) RAS to RAF1, MAPK/ERK kinase (MEK), and ERK, which consequently phosphorylates different effector proteins such as Elk1, Myc, and Rsk, regulating a panel of cellular activities such as proliferation, apoptosis, differentiation, angiogenesis, and metastasis (Fig. 1).

In the canonical ERK signaling pathway, the activating mutation of RAS and amplification of the $R A S / R A F / M E K$ genes are thought to be the main cause of ERK hyperactivation in gastrointestinal (GI) tumors. However, a discrepancy has been found between the widespread activation of ERK (in approximately $90 \%$ of GI tumors) and limited genetic alterations of upstream elements (in $<50 \%$ of GI tumors) [2]. Therefore, how ERK is activated without significant alteration of $R A S, R A F$, and $M E K$ is a major question in the field. This discrepancy also raises a question as to the potential efficacy of inhibitors of RAS/ERK signaling in treating GI tumors.

\section{Synbindin Is a Scaffold Protein of ERK Signaling on the Golgi Apparatus}

We have previously identified synbindin (TRAPPC4), which is a subunit of the transport protein particle (TRAPP) complex responsible for endoplasmic reticulum-Golgi transportation [3], as an important regulator of ERK signaling in GI tumors [4, 5]. Synbindin physically binds to ERK and MEK proteins on the Golgi apparatus, facilitating ERK phosphorylation by MEK. This phosphorylation activates Rsk and Elk1, leading to increased cell proliferation and migration [3]. The synbindin protein contains an atypical PDZ domain and a longin domain [6]. Truncation of the longin domain abolished the interaction between synbindin and ERK [3], and mutation of the ERK DEF domain produced the same result [2]. Therefore, we concluded that the synbindin-longin domain binds the DEF domain of ERK. The expression level of synbindin is strongly correlated with the phosphorylation of ERK protein in gastric cancer tissues [2], which elucidates a noncanonical mechanism for ERK hyperactivation in gastric cancers. Targeting synbindin significantly decreased ERK phosphorylation in a nude mouse model, and tumor growth was also significantly inhibited in the synbindin-inhibited group [3]. These findings highlight the potential therapeutic significance of synbindin in gastric cancer, presumably in complementation with RAS or epidermal growth factor receptor inhibitors.

\section{Synbindin Is Regulated by the OCT1 Transcription Factor}

To clarify the regulatory mechanism of synbindin expression, we searched for transcription factors that may bind to the promoter region of the synbindin gene. We found that the transcription factor OCT1 (POU2F1) had multiple binding sites within the synbindin promoter. This was experimentally confirmed by chromatin immunoprecipitation and luciferase reporter assays. Ectopic expression of OCT1 increased the transcription of synbindin in gastric cancer cells, and knockdown of OCT1 with specific small interfering RNAs caused a substantial decrease in synbindin expression (Fig. 1). The expression of OCT1 and synbindin 


\section{Tumors}

Li et al.: Scaffold Proteins in Gastrointestinal Tumors as a Shortcut to Oncoprotein Activation

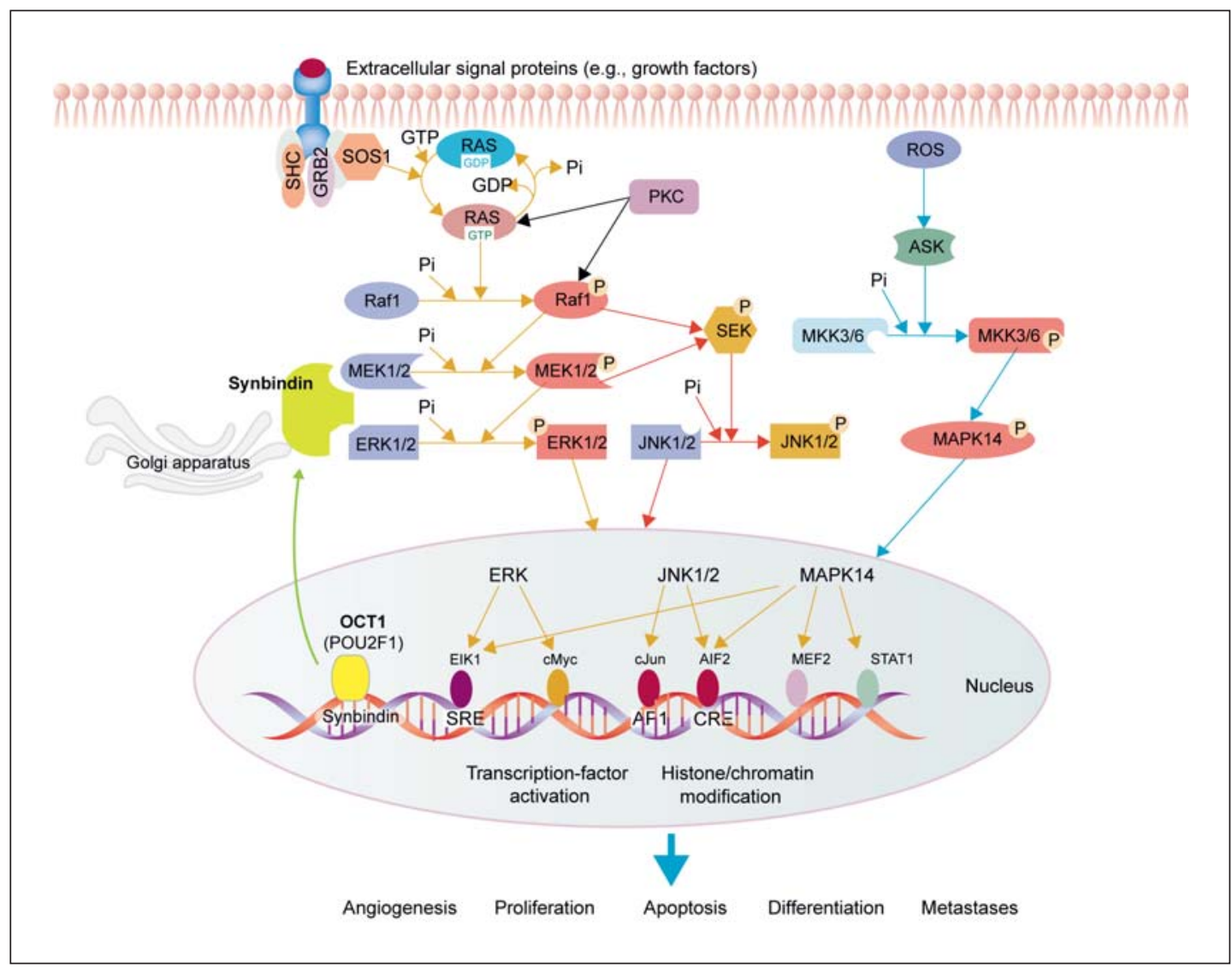

Fig. 1. MAPK pathways and the effects of the OCT1-synbindin axis on ERK signaling. OCT1 transactivates synbindin, which binds both MEK and ERK on the Golgi apparatus, facilitating the activation of Elk1, Myc, and Rsk. Adapted from Fang and Richardson [1], Qian et al. [2], and Kong et al. [3]. P, phosphate; Pi, intracellular organic phosphate.

was strongly correlated in gastric cancer tissues, and OCT1 expression displayed a significant correlation with ERK phosphorylation. Importantly, gene copy amplification, messenger RNA upregulation, and protein overexpression of OCT1 all were significantly associated with poor survival of gastric cancer patients [2]. Moreover, the prognostic significance of OCT1 was confirmed in independent patient cohorts. Although OCT1 has been found to have similar transcription targets as OCT4 (a key factor for generating induced pluripotent stem cells), OCT1 does not replace OCT4 in induced pluripotent stem cell generation $[7,8]$. Instead, OCT1 has been reported to be a determinant of cancer stem cells and somatic stem cells [9]. Interestingly, ERK signaling is also commonly activated in cancer stem cells [10]. These findings support a scenario wherein OCT1 may drive cancer progression by enhancing the ERK signaling pathway. Therefore, synbindin together with its upstream regulator OCT1 may be a promising prognostic biomarker signature and therapeutic target for gastric cancer.

\section{Other Scaffold Proteins in the MAPK Signaling Pathway}

In addition to synbindin, previous studies have revealed multiple crucial scaffold proteins in the MAPK pathway (Table 1). Kinase suppressor of Ras1 (KSR1), known as a classical scaffold protein, exerts significant control over MAPK/ERK signaling. When there is no 
Table 1. Outlines of some scaffold proteins conferring spatiotemporal modulation of the MAPK signaling in GI tumors

\begin{tabular}{|c|c|}
\hline $\begin{array}{l}\text { Scaffold } \\
\text { proteins }\end{array}$ & Sketches \\
\hline Synbindin & $\begin{array}{l}\text { Synbindin binds to ERK and MEK on the Golgi apparatus [3], facilitating ERK phosphorylation } \\
\text { by MEK, which activates Rskand Elk1 [3], leading to increased cell proliferation and migration. } \\
\text { The expression level of synbindin is strongly correlated with the phosphorylation of ERK } \\
\text { protein in gastric cancer tissues [2]. }\end{array}$ \\
\hline KSR1 & $\begin{array}{l}\text { KSR1 constitutively ties to MEK1/2 inactively in cytosolic compartment. Once activated, the } \\
\text { complex relocates to the membrane, interacting with Raf and ERK1/2 and phosphorylating } \\
\text { the cascade [11]. KSR1 has been found to be overexpressed in gastric cancers, and it is sug- } \\
\text { gested that KSR1 is involved in oncogenesis and prognosis, anticancer drug sensitivity, and } \\
\text { chemoresistance in GI tumors }[13,15] \text {. }\end{array}$ \\
\hline IQGAP1 & $\begin{array}{l}\text { IQGAP1 links with B-RAF, MEK1/2, and ERK1/2 through different domains (IQ, WW), re- } \\
\text { sulting in sequential phosphorylation and hyperactivation of ERK1/2 [17]. Studies imply that } \\
\text { the level of IQGAP1 is related to the prognosis in gastric cancer [18] and the treatment } \\
\text { response in rectal adenocarcinomas [19]. }\end{array}$ \\
\hline Paxillin & $\begin{array}{l}\text { Paxillin physically interacts with MEK to induce the activation of ERK when stimulated by } \\
\text { hepatocyte growth factor, exerting control over cell motility [11]. In cancers, paxillin is in- } \\
\text { ferred to be concerned with tumor progression and 5-fluorouracil resistance in colorectal } \\
\text { cancer patients, due to the result that overexpression of paxillin influences the stability of } \\
\text { Bcl-2 via MAPK signaling }[51,52] \text {. }\end{array}$ \\
\hline Sef & $\begin{array}{l}\text { Similar expression to FGF (Sef) regulates the MAPK network through coordination with MEK } \\
\text { to hyperactivate ERK on the Golgi apparatus and prevent ERK shuttling from nuclear to cyto- } \\
\text { solic compartments }[11,53] \text {. The decrease in Sef might cause accumulation of active MEK in } \\
\text { nuclear compartment and thereby upregulation of gene transcription. As a result, Sef might } \\
\text { be an early signal for tumorigenesis [54]. }\end{array}$ \\
\hline
\end{tabular}

stimulus, KSR1 stays inactive in combination with MEK1/2 in cytosolic compartment. Once stimulated by the distinct upstream effector, the conjugate starts to relocate to the membrane and recruit RAF and ERK1/2, and thus the downstream components get activated. Sequentially, the phosphorylated ERKs dimerize and head for the nucleus, modulating transcription [11]. The ERK dimers, simultaneously, phosphorylate B-RAF and KSR1 as a negative feedback, putting an end to the reaction [12]. The expression of KSR1 has been found to vary in different carcinomas [13]. A study observed that compared to the expression in the matched normal tissues, ERK1/2, p-ERK1/2, KSR1, and p-KSR1 were distinctly overexpressed in gastric cancer tissues, in association with TNM stage and the lymph metastasis [14]. Besides, the expressions of ERK1/2, p-ERK1/2, KSR1, and p-KSR1 are significantly related with each other, indicating that KSR1 might play a role in the oncogenesis and progression of gastric neoplasms [15]. Additionally, the KSR1-mediated ERK1/2 signaling pathway is inferred to be somehow associated with anticancer drug sensitivity and chemoresistance in GI tumors $[13,15]$.

IQ motif-containing GTPase activating protein (IQGAP) is another vital scaffold protein participating in the MAPK signaling pathway. The IQGAP family contains three subtypes in human cells, namely IQGAP1, IQGAP2, and IQGAP3 [16]. Among them, IQGAP1 is considered the most important one, as it has been found to take part in numerous cellular activities. Here 
we focus on its role in signaling events causing tumorigenesis. IQGAP1 interacts with three protein kinases (B-RAF, MEK1/2, and ERK1/2) through different domains (IQ, WW), in turn phosphorylating the kinases [17]. Since IQGAP1 can hyperactive ERK, the regulator is presumed to be potentially carcinogenic. Correspondingly, this highly conserved protein has been found to be overexpressed in various carcinomas. Previous research implied that upregulation of IQGAP1 might contribute to poor survival in gastric cancer [18], and both protein expression and localization might impair the treatment response in rectal adenocarcinomas [19].

\section{The Multifaceted Regulation of p53 in GI Tumors}

Although ERK signaling drives the proliferation of cells, the presence of an intact p53 signaling network prevents malignant transformation due to its stringent control of genomic stability [20]. It has long been known that RAS and p53 mutations have strong synergistic effects on tumorigenesis [21, 22]. The function of p53 is commonly lost in cancers, but on a genetic level, the TP53 gene is mutated in approximately half of all cancers [23]. We previously demonstrated that mutant $\mathrm{p} 53$ gains oncogenic function by regulating gastric adenocarcinoma-associated, positive CD44 regulator, long intergenic noncoding (GAPLINC) RNA, and the $C D 44$ oncogene [24]. However, the data suggest that when p53 is not mutated, its inactivation in cancer involves additional regulatory pathways. Such a possible regulator is the E3 ubiquitin ligase MDM2, which has long been recognized as a negative regulator of p53 by targeting p53 for degradation by the proteasome [25, 26]. However, wild-type p53 protein is often found to be highly expressed in cancers where it does not appear to exert a strong tumor-suppressive function [27-29]. How p53 is inactivated in these cases is poorly understood. It has recently been suggested that the function of p53 as a transcription factor is impaired by inefficient acetylation modification [30,31]. However, the factors that control the status of p53 acetylation are unclear, especially in GI tumors.

\section{Scaffold Proteins That Regulate p53 Acetylation and Activation}

Recently, we reported that ArhGAP30 is a determinant of p53 acetylation in colorectal cancer. The ArhGAP30 protein functions as a scaffold protein, binding to the C-terminal of p53 and to P300 acetyltransferase. ArhGAP30 promoted P300-mediated acetylation of p53 K382 residue, and mutation of K382 blocked the effect of ArhGAP30 on p53 function. Importantly, the expression of ArhGAP30 and p53 acetylation was strongly correlated in colorectal cancer tissues, suggesting that ArhGAP30 is a major determinant of p53 acetylation and functional activation in colorectal cancer (Fig. 2) [5]. Of note, we previously reported that some other RhoGAPs (such as ArhGAP11A) may promote the tetramerization of p53 and increase its transcription activity [32]. Therefore, the RhoGAP family seems to have multifaceted roles in p53 function, which are independent of their canonical functions as GTPase activation proteins.

Tribbles 1 (TRIB1), a homologous scaffold protein of Tribbles [33], is a multifunctional regulator of several cellular signaling events. Of note, studies have shed light on its carcinogenic potency for targeting the cancer suppressor p53 [34]. Coordinating with histone deacetylase 1 (HDAC1), TRIB1 facilitates the HDAC1-dependent deacetylation of p53 and thereby suppresses the distinct DNA binding of p53, which consequently results in a decrease in p53-induced cell apoptosis and enhances the incidence of cancer [34]. However, the particular loci where TRIB1 binds p53 and HDAC1 remain to be clarified. Accordingly, the data reveal 


\section{Tumors}

Li et al.: Scaffold Proteins in Gastrointestinal Tumors as a Shortcut to Oncoprotein Activation

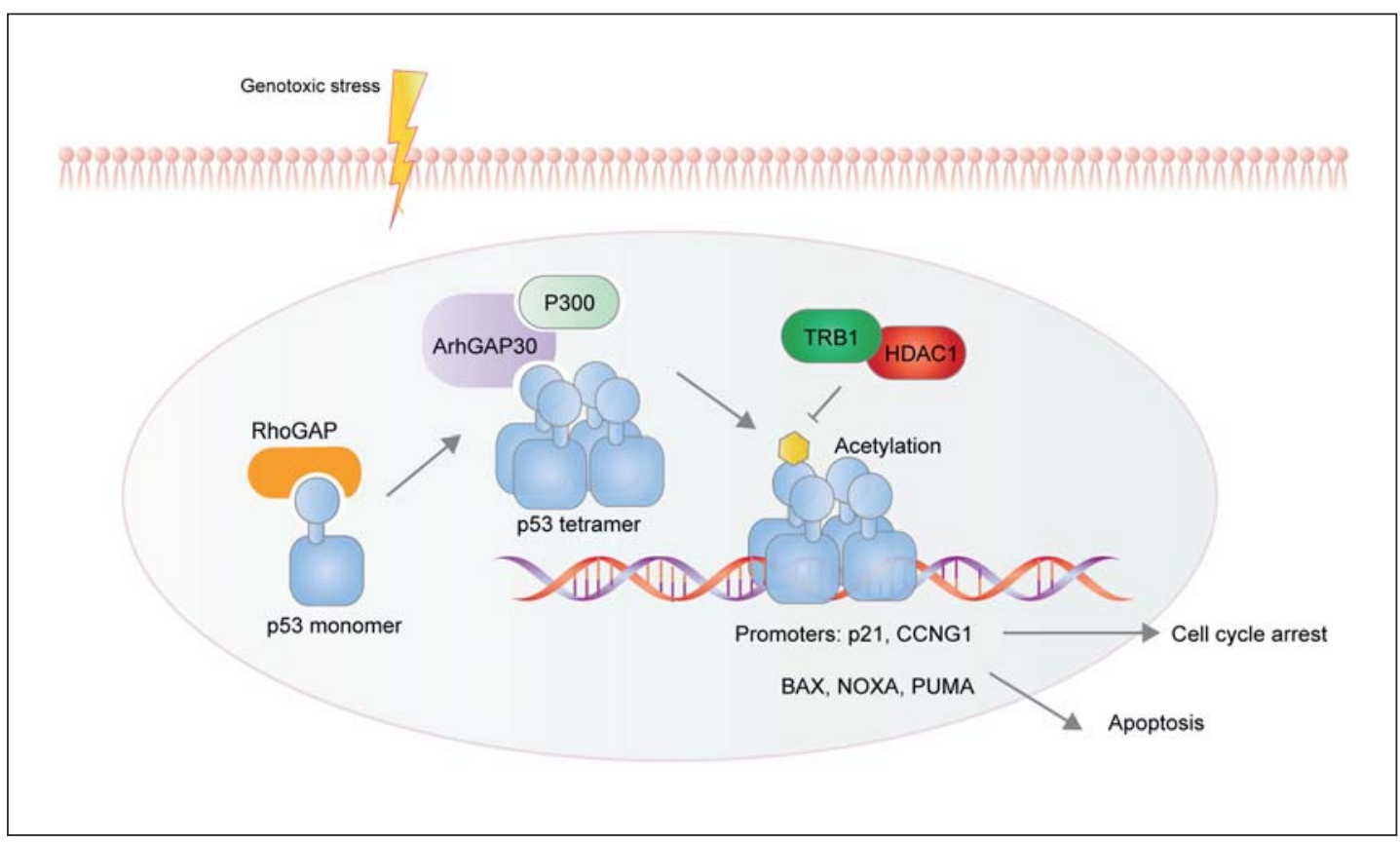

Fig. 2. The ArhGAP30 scaffold protein facilitates P300-mediated acetylation of p53 and its functional activation, and RhoGAP promotes the assembly of p53 monomers into functional tetramers. TRB1 physically ties p53, exerts control over the HDAC1-dependent deacetylation of p53, and thereby suppresses the binding efficacy of the particular DNA and p53. Adapted from Xu et al. [32], Miyajima et al. [34], and Wang et al. [44].

that TRIB1 is upregulated in a variety of carcinomas, including colorectal and esophageal cancer [35]. Given these findings, TRIB1 is probably a novel target for the diagnosis and treatment of GI neoplasms.

\section{Scaffold Proteins That Regulate p53 Ubiquitination}

Wild-type p53 turns inactive via the ubiquitin-proteasome-mediated degradation pathway. This tightly regulated process is mainly associated with MDM2, a major E3 ubiquitin ligase for p53, more exactly with its CTD domain [36] in some conditions. Previous studies have suggested that ubiquitination and degradation of p53 can be upregulated when Twist1 [37], YY1 [38], and gankyrin [39] bind to MDM2, while ARF can be an inhibitor downregulating the ubiquitination of MDM2 [40]. Focal adhesion kinase (FAK) is a multirole tyrosine kinase involved in various cellular events, ranging from cell motility to adhesion and metastasis activities. Strikingly, FAK has been discovered to act as a kinase-independent mechanism, especially as a scaffold protein physically interacting with p53. It is proposed that FAK targets p53 through a distinct region in the N-terminal named FERM, which has three domains termed F1 (binding to p53), F2 (conferring nuclear localization), and F3 (binding to MDM2) [14], while the matched site of p53 is a 7-amino-acid region (amino acids 65-71) in the amino terminal [41]. Both p53 and FAK can shuttle in or out of the nucleus. In the absence of adhesion or in circumstance of cellular stress, FAK runs into the nuclear compartment and interacts with p53 and MDM2; thus, the FAK-p53-MDM2 complex confers ubiquitination and degradation of p53, inducing malignant cell proliferation and apoptosis resistance [14, 42]. However, the intricate relationship between FAK and p53 in cytoplasm is incompletely understood yet. Interestingly, 
Tumors

Li et al.: Scaffold Proteins in Gastrointestinal Tumors as a Shortcut to Oncoprotein Activation

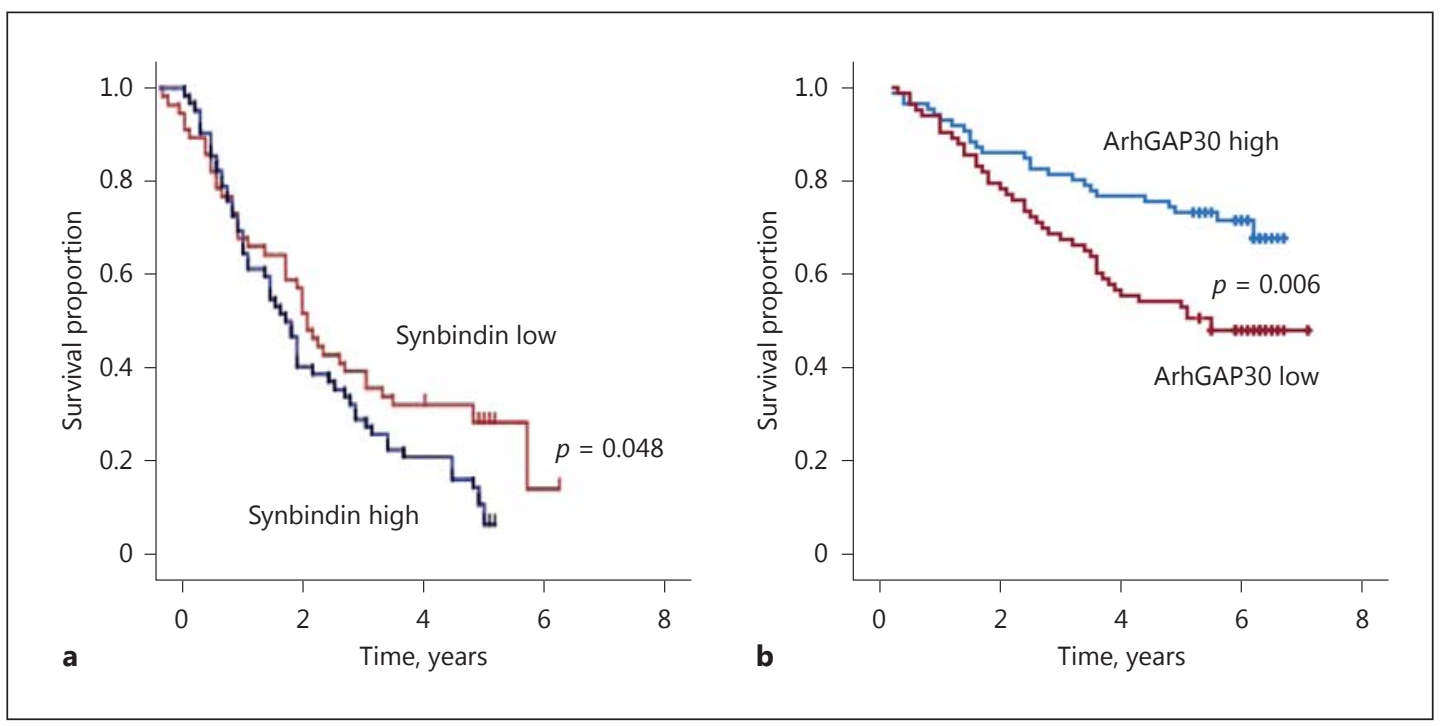

Fig. 3. Prognostic significance of the scaffold proteins synbindin (a) and ArhGAP30 (b) in gastrointestinal tumors. Kaplan-Meier survival analysis was used to compare the prognosis of different patient groups. Adapted from Kong et al. [3] and Wang et al. [44].

p53 can suppress the FAK promoter directly, forming a dynamic feedback loop [43]. Given the fact that the distribution of FAK is abundant in numerous malignant tumors including colon cancer and that the level of FAK is obviously higher in tumors with mutant p53 than in tumors with wild-type p53 [43], the question might be raised as to how to make full use of the bidirectional regulation between FAK and p53, cell survival, and apoptosis and whether it is possible to translate this mechanism into anticancer therapy [42].

\section{Prognostic Significance of Scaffold Proteins}

Since the scaffold proteins have been proved to be of significance in cell proliferation and apoptosis, with MAPK and p53 regulating events, a myriad of relevant research has turned to explore the correlation between the distinct scaffold proteins and tumor prognosis. Studies have revealed that low expression of the ArhGAP30 scaffold protein was associated with poor survival of colorectal cancer patients (Fig. 3), which was supported by independent patient cohorts [44]. The prognostic significance of ArhGAP30 was independent of the American Joint Committee on Cancer (AJCC) tumor stage, suggesting that this biomarker may be used to complement traditional prognostic strategies. We also demonstrated that introduction of the ArhGAP30 gene into tumors could dramatically suppress the growth of colorectal cancer xenografts in nude mice, suggesting that ArhGAP30 could represent a promising therapeutic target. Likewise, synbindin expression was also associated with patient survival in gastric cancer (Fig. 3a).

IQGAP1 has been widely observed in both gastric and colorectal tumors, especially in the deep tissues and invasion fronts of advanced carcinomas [45], which is presumed to be of importance in metastasis and shortened lifespan [46]. Experiments have indicated that tumor susceptibility and aggression may partly rely on the gene dose of IQGAP1 [47], and the absence of IQGAP1 may predict a favorable prognosis [18]. Interestingly, treating tumor-bearing mice with the WW peptide, the only known conserved domain in IQGAP1 bound to ERK, can 
remarkably harm MAPK-mutant neoplasms selectively and reduce resistance to certain anticancer drugs [47]. What is more, it is hypothesized that IQGAP1 is a key to radiochemotherapy resistance [19]. Meanwhile, the overexpression of KSR1 and p-KSR1 is somehow involved with TNM stage and lymph metastases in gastric cancer [14]. Besides, KSR1 offers resistance to multiple antitumor agents, such as cytochalasin $\mathrm{H}$ and tunicamycin, identified through drug screening assays [13], while Ginkgo biloba extract (EGb) 761 has been shown to reduce KSR1-induced chemoresistance by virtue of its antioxidant effect [15]. As for FAK, a meta-analysis accomplished in 2016 [48] declared that upregulation of FAK resulted in worse overall survival (average hazard ratio $=2.073$ ), and in particular, poorer overall survival was observed in gastric cancer (average hazard ratio $=2.073$ ), whereas there seemed to be no correlation between FAK and neoplasm staging. In addition, another report considered that the effect of FAK varied in different phenotypes of gastric cancer, probably due to individual factors [49]. However, a previous analysis revealed no marked prognostic significance of FAK in colon adenocarcinomas [50]. To date, there are few studies providing insight into the clinical significance of TRIB1 in GI tumors. Taken together, the multifunctional scaffold proteins should be attached great importance as promising targets for guiding treatment and estimating prognosis, deserving further study.

\section{Conclusions}

The discovery of scaffold proteins in the ERK and p53 signaling pathways highlights the complexity of cancer cell signaling. Although these scaffold proteins, such as synbindin and ArhGAP30, have opposite effects on cellular phenotypes (pro- and antiproliferation, respectively), they share some common features that deserve our attention: (1) Scaffold proteins do not directly execute posttranslational modifications on their client proteins, but rather catalyze the modification by modulating protein-protein interactions. (2) The expression of scaffold proteins can strongly affect the extent of posttranslational modification in cancer tissues. (3) The expression level of scaffold proteins may be associated with cancer prognosis, and they may be therapeutic targets. Since the expression of many proteins is tissue-specific, it is unclear whether synbindin and ArhGAP30 are also important in the posttranslational modification of ERK and p53 in other cancers and tissue types. It is worthwhile considering that there may be other scaffold proteins for the p53 and ERK pathways in GI tumors.

We are now extending our research by constructing a gene knockout model of synbindin. In this new system, the roles of synbindin in cancer development can be determined at higher resolution. The effects of synbindin disruption on gene expression and protein posttranslational modifications will be examined by microarray and mass spectrometry. Through this work, we hope to understand the roles of scaffold proteins in a more comprehensive manner.

\section{Acknowledgments}

This project was supported by grants from National Natural Science Foundation of China (81572326, 81322036, 30971330, 31371420, 81320108024, 81000861, 81421001, and 81272383), the Top-Notch Young Talents Program of China (ZTZ2015-48), the Shanghai “Oriental Scholars" project (2013XJ), and Shanghai Municipal Education Commission-Gaofeng Clinical Medicine Grant Support.

\section{Disclosure Statement}

All authors declare that they have no conflict of interest regarding this work. 


\section{References}

1 Fang JY, Richardson BC: The MAPK signalling pathways and colorectal cancer. Lancet Oncol 2005;6:322-327.

2 Qian J, et al: OCT1 is a determinant of synbindin-related ERK signalling with independent prognostic significance in gastric cancer. Gut 2015;64:37-48.

3 Kong X, et al: Synbindin in extracellular signal-regulated protein kinase spatial regulation and gastric cancer aggressiveness. J Natl Cancer Inst 2013;105:1738-1749.

4 Weng YR, et al: The role of ERK2 in colorectal carcinogenesis is partly regulated by TRAPPC4. Mol Carcinog 2014;53(suppl 1):E72-E84.

5 Zhao SL, et al: TRAPPC4-ERK2 interaction activates ERK1/2, modulates its nuclear localization and regulates proliferation and apoptosis of colorectal cancer cells. PLoS One 2011;6:e23262.

6 Fan S, et al: Solution structure of synbindin atypical PDZ domain and interaction with syndecan-2. Protein Pept Lett 2009;16:189-195.

7 Esch D, et al: A unique Oct4 interface is crucial for reprogramming to pluripotency. Nat Cell Biol 2013;15: 295-301.

8 Kang J, Shakya A, Tantin D: Stem cells, stress, metabolism and cancer: a drama in two Octs. Trends Biochem Sci 2009;34:491-499.

9 Maddox J, et al: Transcription factor Oct1 is a somatic and cancer stem cell determinant. PLoS Genet 2012; 8:e1003048.

10 Khurana SS, et al: The hyaluronic acid receptor CD44 coordinates normal and metaplastic gastric epithelial progenitor cell proliferation. J Biol Chem 2013;288:16085-16097.

11 Witzel F, Maddison L, Bluthgen N: How scaffolds shape MAPK signaling: what we know and opportunities for systems approaches. Front Physiol 2012;3:475.

12 McKay MM, Ritt DA, Morrison DK: Signaling dynamics of the KSR1 scaffold complex. Proc Natl Acad Sci USA 2009;106:11022-11027.

13 Stoeger SM, Cowan KH: Characterization of kinase suppressor of Ras-1 expression and anticancer drug sensitivity in human cancer cell lines. Cancer Chemother Pharmacol 2009;63:807-818.

14 Lim ST, et al: Nuclear FAK promotes cell proliferation and survival through FERM-enhanced p53 degradation. Mol Cell 2008;29:9-22.

15 Liu SQ, et al: Ginkgo biloba extract enhances chemotherapy sensitivity and reverses chemoresistance through suppression of the KSR1-mediated ERK1/2 pathway in gastric cancer cells. Oncol Rep 2015;33:2871-2882.

16 White CD, Brown MD, Sacks DB: IQGAPs in cancer: a family of scaffold proteins underlying tumorigenesis. FEBS Lett 2009;583:1817-1824.

17 Abel AM, et al: IQGAP1: insights into the function of a molecular puppeteer. Mol Immunol 2015;65:336-349.

18 Walch A, et al: Combined analysis of Rac1, IQGAP1, Tiam1 and E-cadherin expression in gastric cancer. Mod Pathol 2008;21:544-552.

19 Holck S, et al: IQGAP1 in rectal adenocarcinomas: localization and protein expression before and after radiochemotherapy. Cancer Lett 2015;356(2 Pt B):556-560.

$20 \mathrm{Xu}$ J, et al: Gain of function of mutant p53 by coaggregation with multiple tumor suppressors. Nat Chem Biol 2011;7:285-295.

21 Fukasawa K, Vande Woude GF: Synergy between the Mos/mitogen-activated protein kinase pathway and loss of p53 function in transformation and chromosome instability. Mol Cell Biol 1997;17:506-518.

22 Hsieh JS, et al: APC, K-ras, and p53 gene mutations in colorectal cancer patients: correlation to clinicopathologic features and postoperative surveillance. Am Surg 2005;71:336-343.

23 Kandoth C, et al: Mutational landscape and significance across 12 major cancer types. Nature 2013;502:333339.

$24 \mathrm{Hu}$ Y, et al: Long noncoding RNA GAPLINC regulates CD44-dependent cell invasiveness and associates with poor prognosis of gastric cancer. Cancer Res 2014;74:6890-6902.

25 Oliner JD, et al: Oncoprotein MDM2 conceals the activation domain of tumour suppressor p53. Nature 1993; 362:857-860.

26 Haupt Y, et al: Mdm2 promotes the rapid degradation of p53. Nature 1997;387:296-299.

27 Pardo FS, et al: Mutant, wild type, or overall p53 expression: freedom from clinical progression in tumours of astrocytic lineage. Br J Cancer 2004;91:1678-1686.

28 Houben R, et al: High-level expression of wild-type p53 in melanoma cells is frequently associated with inactivity in p53 reporter gene assays. PLoS One 2011;6:e22096.

$29 \mathrm{Xu}$ J, et al: Unequal prognostic potentials of p53 gain-of-function mutations in human cancers associate with drug-metabolizing activity. Cell Death Dis 2014;5:e1108.

30 Munoz-Fontela C, et al: Acetylation is indispensable for p53 antiviral activity. Cell Cycle 2011;10:3701-3705.

31 Brooks CL, Gu W: The impact of acetylation and deacetylation on the p53 pathway. Protein Cell 2011;2:456462.

$32 \mathrm{Xu} \mathrm{J}$, et al: RhoGAPs attenuate cell proliferation by direct interaction with p53 tetramerization domain. Cell Rep 2013;3:1526-1538.

33 Mata J, Curado S, Ephrussi A, Rørth P: Tribbles coordinates mitosis and morphogenesis in Drosophila by regulating string/CDC25 proteolysis. Cell 2000;101:511-522. 
34 Miyajima C, Inoue Y, Hayashi H: Pseudokinase Tribbles 1 (TRB1) negatively regulates tumor-suppressor activity of p53 through p53 deacetylation. Biol Pharm Bull 2015;38:618-624.

35 Liang JW, et al: Analysis of genomic aberrations associated with the clinicopathological parameters of rectal cancer by array-based comparative genomic hybridization. Oncol Rep 2013;29:1827-1834.

36 Poyurovsky MV, et al: The C terminus of p53 binds the N-terminal domain of MDM2. Nat Struct Mol Biol 2010; 17:982-989.

37 Piccinin S, et al: A "twist box" code of p53 inactivation: twist box: p53 interaction promotes p53 degradation. Cancer Cell 2012;22:404-415.

38 Sui G, et al: Yin Yang 1 is a negative regulator of p53. Cell 2004;117:859-872.

39 Higashitsuji H, et al: The oncoprotein gankyrin binds to MDM2/HDM2, enhancing ubiquitylation and degradation of p53. Cancer Cell 2005;8:75-87.

40 Xirodimas D, et al: Different effects of p14ARF on the levels of ubiquitinated p53 and Mdm2 in vivo. Oncogene 2001;20:4972-4983.

41 Golubovskaya VM, et al: The 7-amino-acid site in the proline-rich region of the N-terminal domain of p53 is involved in the interaction with FAK and is critical for p53 functioning. Biochem J 2008;411:151-160.

42 Cance WG, Golubovskaya VM: Focal adhesion kinase versus p53: apoptosis or survival? Sci Signal 2008; 1:pe22.

43 Golubovskaya VM, et al: p53 regulates FAK expression in human tumor cells. Mol Carcinog 2008;47:373-382.

44 Wang J, et al: ArhGAP30 promotes p53 acetylation and function in colorectal cancer. Nat Commun 2014;5: 4735.

45 Nabeshima K, et al: Immunohistochemical analysis of IQGAP1 expression in human colorectal carcinomas: its overexpression in carcinomas and association with invasion fronts. Cancer Lett 2002;176:101-109.

46 Hayashi H, et al: Overexpression of IQGAP1 in advanced colorectal cancer correlates with poor prognosiscritical role in tumor invasion. Int J Cancer 2010;126:2563-2574.

47 Jameson KL, et al: IQGAP1 scaffold-kinase interaction blockade selectively targets RAS-MAP kinase-driven tumors. Nat Med 2013;19:626-630.

48 Zeng XQ, et al: Prognostic value of focal adhesion kinase (FAK) in human solid carcinomas: a meta-analysis. PLoS One 2016;11:e0162666.

49 Giaginis CT, et al: Expression and clinical significance of focal adhesion kinase in the two distinct histological types, intestinal and diffuse, of human gastric adenocarcinoma. Pathol Oncol Res 2009;15:173-181.

50 Theocharis SE, et al: Focal adhesion kinase expression is not a prognostic predictor in colon adenocarcinoma patients. Eur J Surg Oncol 2003;29:571-574.

51 Huang CC, et al: Paxillin promotes colorectal tumor invasion and poor patient outcomes via ERK-mediated stabilization of Bcl-2 protein by phosphorylation at Serine 87. Oncotarget 2015;6:8698-8708.

52 Wu DW, et al: Bcl-2 stabilization by paxillin confers 5 -fluorouracil resistance in colorectal cancer. Cell Death Differ 2015;22:779-789.

53 Brown MD, Sacks DB: Protein scaffolds in MAP kinase signalling. Cell Signal 2009;21:462-469.

54 Duhamel S, et al: Sef downregulation by Ras causes MEK1/2 to become aberrantly nuclear localized leading to polyploidy and neoplastic transformation. Cancer Res 2012;72:626-635. 